\title{
Effect of Drying Temperature on Sensory and Flavor of Mint
}

\author{
Xiaoyan Zhao ${ }^{\mathrm{a}}$, Chao Zhang ${ }^{\mathrm{b}}$, Yubin $\mathrm{Wang}^{\mathrm{c}}$, Yue Ma ${ }^{\mathrm{d}}$
}

Beijing Vegetable Research Center, Beijing Academy of Agriculture and Forestry Sciences; Beijing Key Laboratory of Fruits and Vegetable Storage and Processing; Key Laboratory of Biology and Genetic Improvement of Horticultural Crops (North China), Ministry of Agriculture; Key Laboratory of Urban Agriculture (North), Ministry of Agriculture, 9 Shuguanghuayuan Road, Haidian District, Beijing, China

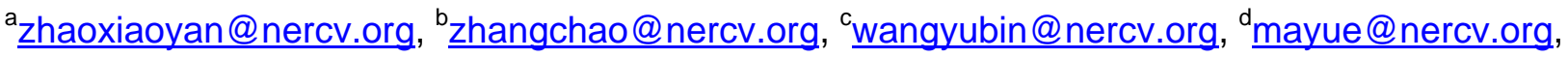

Keywords: fresh cut, mint, drying, flavor, sensory

Abstract. The effect of drying temperature on sensory and flavor of dehydrated mint was evaluated. The color and appearance of the dehydrated mint were affected by the drying temperature. A lower temperature, such as 55 and $65{ }^{\circ} \mathrm{C}$, preserved the greenness and avoided the browning of the dehydrated mint. The flavor of the dehydrated mint was similar after each drying temperature. Hence, a lower drying temperature was a better option for the processing of the dehydrated mint.

\section{Introduction}

Mint (Mentha spicata L.) has a wide range of uses such as cooking as a spicy herb, aromatherapy as a fragrance, manufacturing of candy, toothpaste and gum[1]. Mint leaves have presented antioxidant[2], anti-inflammatory[3], and antibacterial activities[4], and antiallergic activity[5].

The demand of the dehydrated mint enhances quickly in the past decade due to its wide applications. Drying temperature is an important factor influencing the final quality of the products [6]. However, the evaluation of the sensory and flavor of the mint was neglected during the drying process to the best of our knowledge. Hence, the effect of drying temperature on sensory and flavor of mint was evaluated in the current study. The flavor and appearance of the mint dried at a different temperature was compared.

\section{Material and Methods}

Dehydration of mint. Mint was picked from our Tongzhou farm (Tongzhou District Beijing, 2014) and was stored at $4{ }^{\circ} \mathrm{C}$ before use. The mint was washed by the tap water at $4{ }^{\circ} \mathrm{C}$ to remove the soil and inclusions. The washed mint was heated at 55, 65, 75, 85, and $95{ }^{\circ} \mathrm{C}$ in a air-circulated oven respectively. The moisture content of $7.0 \%$ was the termination point for each drying. During each drying, about 7 samples were picked up for the quality determination. The fresh mint was designated as the control.

Color determination. The sample was powdered and measured by a reflective mode in a $0.5 \mathrm{~cm}$ cuvette, followed the recently reported method[7]. The color of samples was assessed in a LAB space with the dimension $\boldsymbol{L}^{*}$ for lightness and $\boldsymbol{a}^{*}$ and $\boldsymbol{b}^{*}$ for the color-opponent dimensions by a spectrophotometer (CM3700d, Konica Minolta Sensing INC., Japan). Specifically, the value $L^{*}$ represents the lightness of the color $\left(\boldsymbol{L}^{*}=0\right.$ yields black and $\boldsymbol{L}^{*}=100$ indicates diffuse white); the negative value $\boldsymbol{a}^{*}$ indicates green while positive values indicate magenta; the negative value $\boldsymbol{b}^{*}$ indicates blue and positive values indicate yellow. The fresh mint was measured as the control.

Flavor comparison. The flavor of the samples was compared by an electronic nose PEN2 (Airsense Analytics GmbH, Schwerin, Germany), followed the recently method [8]. The electronic nose was turned on for $30 \mathrm{~min}$ and flushed the testing system for $180 \mathrm{~s}$. The sample of $2 \mathrm{ml}$ was put in the testing tube. And then the electronic sensor was put into the testing tube to collect the results for $60 \mathrm{~s}$. The response of the sensor in $48 \sim 52 \mathrm{~s}$ were evaluated by a principal component analysis. The fresh mint was designated as the control.

Statistical Analysis. Analysis of variance (ANOVA) was used to compare mean differences of the results. If the differences in mean existed, multiple comparisons were performed using Duncan's 
Multiple Range Test. All analysis was conducted using SPSS for Window Version 19. All experiments were done in triplicates or more.

\section{Results and Discussion}

Effect of drying temperature on color of mint. The color of the mint dehydrated to the moisture content of $7.0 \%$ at a different temperature was compared with the color of the control (Figure 1). The color of the mint was expressed by the LAB system. the value $L^{*}$ represents the lightness of the color ( $\boldsymbol{L}^{*}=0$ yields black and $\boldsymbol{L}^{*}=100$ indicates diffuse white) $[9,10]$. The $\boldsymbol{L}^{*}$ of the dehydrated mint was enhanced significantly after drying at each temperature. Moreover, the $\boldsymbol{L}^{*}$ value was enhanced with the drying temperature. The $L^{*}$ value of the mint dried at $95{ }^{\circ} \mathrm{C}$ was significant higher than that dried at the other temperature.

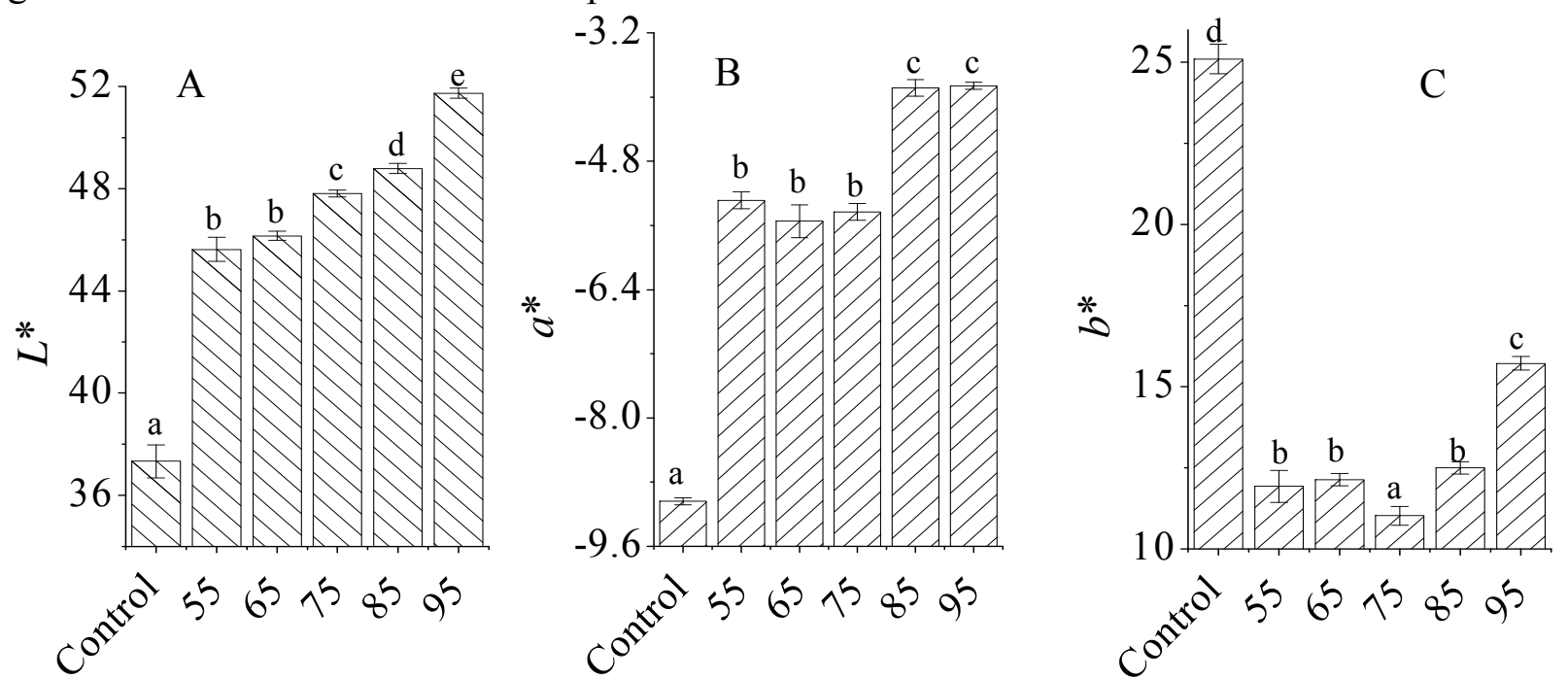

Figure 1 Effect of drying temperature on $L^{*}$ value (A), $a^{*}$ value $(\mathrm{B})$ and

$b^{*}$ value $(\mathrm{C})$ of dehydrated mint

The negative $\boldsymbol{a}^{*}$ value indicates green while positive values indicate magenta [11]. The $a^{*}$ value is closely related to the greenness of a sample. The greenness of the mint was mainly came from the chlorophyll. Consequently, the $a^{*}$ value was an indicator of the chlorophyll content. The chlorophyll content is decrease due to its degradation. The chlorophyll degradation of the spinach [12] and kiwifruit puree [13] is found to follow the first-order kinetics. The $a^{*}$ value of the dehydrated mint was enhanced after drying. The $a^{*}$ value of the dehydrated mint dried at 55, 65 and $75{ }^{\circ} \mathrm{C}$ was significant higher than that of the control, while that was significantly lower than that of that dried at 85 and $95{ }^{\circ} \mathrm{C}$. Consequently, the greenness of the mint dried at 85 and $95{ }^{\circ} \mathrm{C}$ was lighter than that dried at the other temperature. The phenomenon proved that a lower temperature preserved the original color of the dehydrated mint.

The negative value $\boldsymbol{b}^{*}$ indicates blue and positive values indicate yellow [11]. The $b^{*}$ value was reduced after the drying treatments. The $b^{*}$ value of the mint dried at $95{ }^{\circ} \mathrm{C}$ was significant higher than dried at the other temperature.

Effect of drying temperature on flavor of mint. The effect of drying temperature on flavor of mint is shown in Figure 2. The principal component analysis showed that the flavor of the samples was mainly contributed by the main component 1 and main component 2 . The variance of the main component 1 and main component 2 was $95.95 \%$ and $3.84 \%$, respectively. The sum of the main component 1 and 2 was $99.79 \%$, which was effective to reflect the flavor difference of the mint. Each drying temperature leaded to a significant difference compared with the flavor of the control. Remarkably, the flavor of each mint was similar. Consequently, the drying temperature showed no influence on the flavor of the dehydrated mint. Being different to our results, the flavor of spearmint and peppermint is affected be the drying temperature $[14,15]$. 


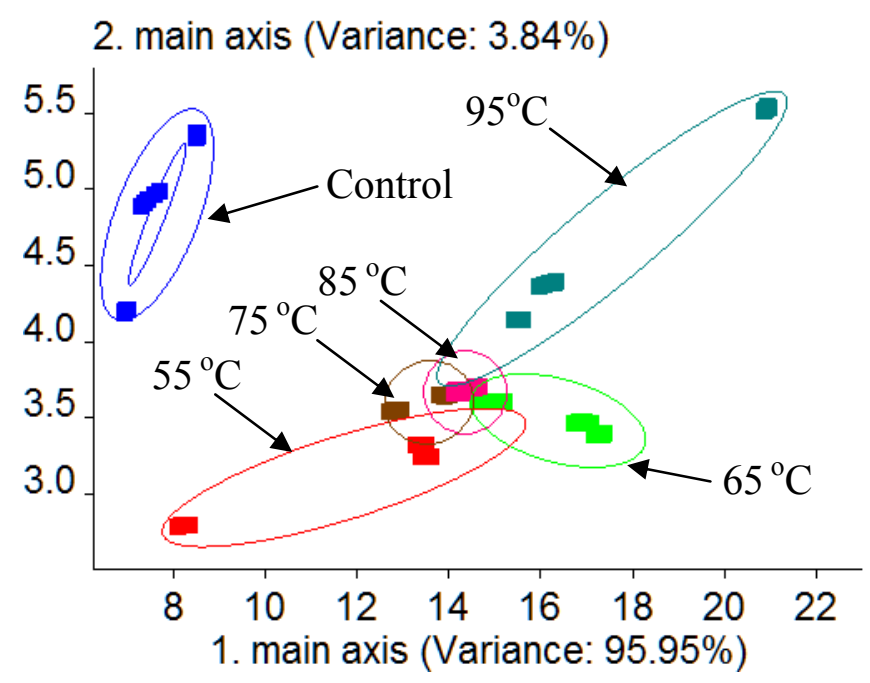

Figure 2 Effect of drying temperature on flavor of dehydrated mint

Effect of dehydration on appearance of mint. The effect of drying temperature on appearance of mint is shown in Figure 3. The color of the dehydrated mint was a little yellow to the color of the fresh mint. Moreover, the drying temperature also affected the greenness and yellowness of the mint. The mint dehydrated at 55,65 and $75{ }^{\circ} \mathrm{C}$ showed more greenness, while that dehydrated at the other temperature showed more yellowness. The phenomenon was also been validated by the color evaluation. On the other hand, a higher temperature leaded to a browning of the product [16]. Hence, a lower drying temperature was preferred from the appearance of the dehydrated mint.

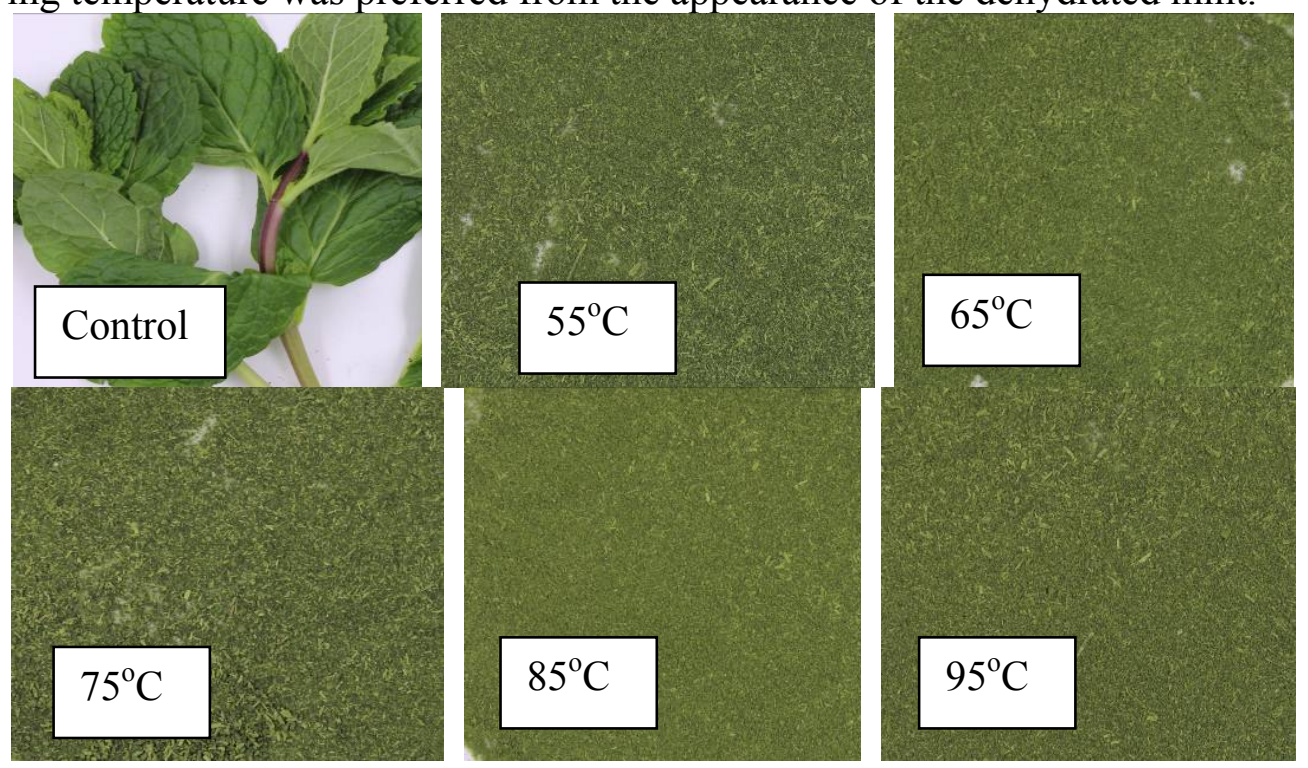

Figure 3 Effect of drying temperature on appearance of dehydrated mint

\section{Conclusions}

The color and appearance of the dehydrated were affected by the drying temperature. A lower temperature, such as 55 and $65{ }^{\circ} \mathrm{C}$ preserved the greenness and avoided the browning of the dehydrated mint. The flavor of the dehydrated mint was similar after each drying temperature. Hence, a lower drying temperature was a better option for the processing of the dehydrated mint.

\section{Acknowledgements}

The authors are grateful to financial support of the earmarked fund for Modern Agro-industry Technology Research System (CARS-26-22 \& CARS -25), Beijing Academy of Agricultural and 
Forestry Sciences, New Discipline Breeding (KJCX20140204) and Beijing Key Laboratory of Fruits and Vegetable Storage and Processing (Z141105004414037).

\section{References}

[1] O. T. Asekun, D. S. Grierson, A. J. Afolayan: Food Chem. Vol 101 (2007) 995.

[2] S. R. Kanatt, R. Chander, A. Sharma: Food Chem. Vol 100 (2007) 451.

[3] P. Arumugam, N. G. Priya, M. Subathra, A. Ramesh: Environm. Toxicol. Pharmacol. Vol 26 (2008) 92.

[4] C. Tassou, K. Koutsoumanis, G. J. E. Nychas: Food Res. Int. Vol 33 (2000) 273.

[5] A. Sato, H. Tamura: Fitoterapia Vol 102 (2015) 74.

[6] S. Tarhan, I. Telci, M. T. Tuncay, H. Polatci: Industrial Crops Product. Vol 32 (2010) 420.

[7] J. Tian, X. Zeng, S. Zhang, Y. Wang, P. Zhang, A. Lu, X. Peng: Industrial Crops Product. Vol $59(2014) 69$.

[8] M. Laureati, S. Buratti, A. Bassoli, G. Borgonovo, E. Pagliarini: Food Res. Int. Vol 43 (2010) 959.

[9] S. A. Osmani, E. H. Hansen, C. Malien-Aubert, C. E. Olsen, S. Bak, B. L. Mller: J. Agric. Food Chem. Vol 57 (2009) 3149.

[10]T. P. Labuza: J. Food Sci. Vol 51 (1986) ii.

[11]L. Rolle, S. Guidoni: J. Int. Des Sciences De La Vigne Et Du Vin Vol 41 (2007) 193.

[12]F. M. Lajolo, U. M. Lanfer Marquez: J. Food Sci. Vol 47 (1982) 1995.

[13]M. Benlloch-Tinoco, A. Kaulmann, J. Corte-Real, D. Rodrigo, N. Martinez-Navarrete, T. Bohn: Food Chem. Vol 187 (2015) 254.

[14]R. Baranauskienè, E. Bylaite, J. Žukauskaite, P. R. Venskutonis: J. Agric. Food Chem. Vol 55 (2007) 3027.

[15]M. C. Díaz-Maroto, M. S. Pérez-Coello, M. A. G. Viñas, M. D. Cabezudo: J. Agric. Food Chem. Vol 51 (2003) 1265.

[16]T. Imaizumi, T. Orikasa, S. Morifusa, L. V. Man, Y. Muramatsu, S. Koide, T. Uchino, F. Tanaka, D. Hamanaka, A. Tagawa: Eng. Agric., Environm. Food Vol 8 (2015) 1. 